\title{
Bidirectional Promoter-Based CRISPR-Cas9 Systems for Plant Genome Editing
}

\author{
Qiurong Ren ${ }^{1+}$, Zhaohui Zhong ${ }^{1 \dagger}$, Yan Wang ${ }^{1}$, Qi You ${ }^{2,3}$, Qian Li ${ }^{1}$, Mingzhu Yuan ${ }^{1}$, \\ Yao He ${ }^{1}$, Caiyan $\mathrm{Qi}^{1}{ }^{1}, \mathrm{Xu} \mathrm{Tang}^{1}{ }^{1}$, Xuelian Zheng ${ }^{1}$, Tao Zhang ${ }^{2,3 *}$, Yiping Qi ${ }^{4,5 *}$ and \\ Yong Zhang ${ }^{1,2 *}$
}

\begin{abstract}
${ }^{1}$ Department of Biotechnology, School of Life Sciences and Technology, Center for Informational Biology, University of Electronic Science and Technology of China, Chengdu, China, ${ }^{2}$ Jiangsu Key Laboratory of Crop Genetics and Physiology, Jiangsu Co-Innovation Center for Modern Production Technology of Grain Crops, Jiangsu Key Laboratory of Crop Genomics and Molecular Breeding, Agricultural College of Yangzhou University, Yangzhou, China, ${ }^{3}$ Key Laboratory of Plant Functional Genomics of the Ministry of Education, Joint International Research Laboratory of Agriculture and Agri-Product Safety of the Ministry of Education, Yangzhou University, Yangzhou, China, ${ }^{4}$ Department of Plant Science and Landscape Architecture, University of Maryland, College Park, MD, United States, ${ }^{5}$ Institute for Bioscience and Biotechnology Research, University of Maryland, Rockville, MD, United States
\end{abstract}

CRISPR-Cas systems can be expressed in multiple ways, with different capabilities regarding tissue-specific expression, efficiency, and expression levels. Thus far, three expression strategies have been demonstrated in plants: mixed dual promoter systems, dual Pol II promoter systems, and single transcript unit (STU) systems. We explored a fourth strategy to express CRISPR-Cas9 in the model and crop plant, rice, where a bidirectional promoter (BiP) is used to express Cas9 and single guide RNA (sgRNA) in opposite directions. We first tested an engineered BiP system based on double-mini 35 S promoter and an Arabidopsis enhancer, which resulted in $20.7 \%$ and $52.9 \%$ genome editing efficiencies at two target sites in T0 stable transgenic rice plants. We further improved the BiP system drastically by using a rice endogenous BiP, OsBiP1. The endogenous BiP expression system had higher expression strength and led to 75.9-93.3\% genome editing efficiencies in rice T0 generation, when the sgRNAs were processed by either tRNA or Csy4. We provided a proof-of-concept study of applying BiP systems for expressing two-component CRISPR-Cas9 genome editing reagents in rice. Our work could promote future research and adoption of BiP systems for CRISPR-Casbased genome engineering in plants.

Keywords: CRISPR-Cas9, plant genome editing, rice, bidirectional promoter, enhancer

\section{INTRODUCTION}

CRISPR-Cas9 and Cas12a (formerly Cpf1) are widely used sequence-specific nucleases (SSNs) for plant genome editing (Jiang et al., 2013; Li et al., 2013; Nekrasov et al., 2013; Fauser et al., 2014; Endo et al., 2016; Xu et al., 2016; Begemann et al., 2017; Tang et al., 2017; Zhou et al., 2017; Lowder et al., 2018; Tang et al., 2018; Zhong et al., 2018; Zhong et al., 2019; Zhou et al., 2019). Unlike meganucleases, zinc finger nucleases (ZFNs), and TAL effector nucleases (TALENs), a CRISPRCas system relies on a single guide RNA (sgRNA, for Cas9) or CRISPR RNA (crRNA, for Cas12a) for DNA targeting, bypassing protein engineering. This easiness has made CRISPR-Cas systems as 
top SSN choices for plant reverse genetics and accelerated crop breeding. While it is possible to deliver the Cas protein and the guide RNA as preformed ribonucleoprotein (RNP) complexes (Woo et al., 2015; Svitashev et al., 2016; Liang et al., 2017; Andersson et al., 2018), plant applications have largely relied on Agrobacterium mediated T-DNA transformation where Cas9/Cas12a and guide RNA expression cassettes are packaged into a T-DNA vector. Conventionally, a Cas gene is expressed by a Pol II promoter and a guide RNA is expressed by a Pol III promoter, and this system is termed as a mixed dual promoter system. However, Pol III promoters, such as U6 or U3, cannot match the expression strength of some strong Pol II promoters, which limits the overall genome editing efficiency in plants (Tang et al., 2016; Cermak et al., 2017; Mikami et al., 2017; Tang et al., 2019). Further, Pol III promoters are only suitable for expression of relatively short transcripts, which prevents the use of a single Pol III promoter to effectively express multiple sgRNAs for multiplexed genome editing, an important feature and advantage of CRISPR-Cas technologies.

To utilize Pol II promoters for guide RNA expression, two novel CRISPR-Cas expression systems have been developed in recent years. The first system, a dual Pol II promoter system, utilizes two separate Pol II promoter-terminator cassettes to express the Cas gene and the guide RNA. This system has been demonstrated for efficient plant genome editing with Cas9 (Cermak et al., 2017) and Cas12a (Tang et al., 2017; Zhong et al., 2018). However, repeated use of the same promoter may have a risk of gene silencing. Using two different Pol II promoters for Cas and guide RNAs may address this potential problem. Use of two separate promoters, however, adds to the length of the final DNA expression constructs. The second system, single transcript unit (STU), utilizes only one promoter to express the Cas gene and the guide RNAs for plant genome editing, as demonstrated for CRISPR-Cas9 (Tang et al., 2016; Mikami et al., 2017; Zhou et al., 2017; Tang et al., 2019) and CRISPR-Cas12a (Zhong et al., 2018; Tang et al., 2019). While STU is very compact, the expression system may not be optimal for the CRISPR-Cas system. This is because the same amount of Cas-sgRNA STU mRNAs is transcribed, yet it takes another protein translation step to generate Cas proteins. It hence may not be possible to achieve 1:1 molar ratio of Cas protein and guide RNA in vivo based on a STU expression system.

It would be useful to develop bidirectional promoter ( $\mathrm{BiP})$ systems for CRISPR-Cas expression. In this case, it only requires one promoter to express both Cas and guide RNAs (just as in a STU system). However, expression of either component can be independently fine-tuned with the use of different 3 '-UTR or/ and terminators (as with a dual Pol II system). Recently, a BiP CRISPR-Cas9 system, coupled with ribozyme-based sgRNA processing, was successfully developed for efficient genome editing in Oleaginous Microalga (Poliner et al., 2018). In this study, we explored BiP strategies for the expression of CRISPRCas9 for genome editing and sought to prove the concept in rice, which is a model plant and a major crop. We tested an engineered $\mathrm{BiP}$ system as well as a plant endogenous $\mathrm{BiP}$ system. Our study suggests promising applications of $\mathrm{BiP}$ systems for efficient expression of CRISPR-Cas systems in plant genome editing.

\section{MATERIALS AND METHODS}

\section{Vector Construction}

The BiP CRISPR-Cas9 system plasmids were constructed using pTX152 (p35S::Hyg::35S T + p35S::Cas9::Hsp T) (Tang et al., 2016; Zhou et al., 2017) as a vector backbone. To construct Cas9-tRNA intermediate cloning vector (pGEL038), tRNA::ccdB::sgRNA::tRNA and Cas9 fragment were amplified from pGEL031 (Tang et al., 2019), and Ocs terminator was amplified from pZHY933 (Zhou et al., 2019). Next, these three fragments were cloned in between the AscI and Sbfl sites of pTX152 (Tang et al., 2016; Zhou et al., 2017) by Gibson assembly. To construct Cas9-Csy4 intermediate cloning vector (pGEL039), Csy4 cleavage site::ccdb::gRNA scaffold::Csy4 cleavage site fragment and the Csy4-P2A fragment were amplified from pGEL031 (Tang et al., 2019) and then cloned in between the $K p n I$ and Sbfl sites of the Cas9-tRNA plasmid by Gibson assembly. To construct the mini 35s-Cas9-Csy4 system (pGEL050), two CaMV 35S minimal promoters were synthesized and further cloned into the SpeI and Sbfl sites of Cas9-Csy4 system by T4 ligase. To create the mini 35s-enhancer-Cas9-Csy4 system (pGEL051), the Arabidopsis enhancer (additional data: Figure S4) (Zhu et al., 2015; Zhang et al., 2016) was amplified from Arabidopsis Col-0, cloned into XbaI and PvuII-linearized pGEL050 plasmids by T4 ligase. To generate the OsBiP1-Cas9-tRNA system (pGEL052) and OsBiP1-Cas9-Csy4 system (pGEL053), the OsBiP1 promoter fragment (additional data: Figure S5) (Wang et al., 2016) was amplified from Oryza sativa Geng/Japonica cultivar Nipponbare and cloned into pGEL038 and pGEL039 by Gibson assembly. For creating nuclease expression vector, sgRNAs were synthesized as duplexed oligonucleotides (Table S1). Oligos were annealed into BsaI-linearized Cas9 vectors by Golden Gate cloning. The fragments of mini 35s-green-fluorescent protein (GFP) (pGEL056), mini 35s-enhancer-GFP (pGEL057), and OsBiP1-GFP (pGEL058) were respectively amplified from pGEL050, pGEL051, and pGEL052 and cloned into ZmUbi-GFP (pGEL055) by Gibson assembly. The vectors generated in this study are available at Yong Zhang Lab upon request.

\section{Rice Protoplast Transformation}

The $O$. sativa Geng/Japonica cultivar Nipponbare was used in this study. Rice protoplast isolation and transformation with T-DNA vectors were performed according to our previously published protocols (Shan et al., 2013; Zhang et al., 2013; Zheng et al., 2016). Rice seedlings were grown at $28^{\circ} \mathrm{C}$ in the dark for 12 days. Thirty to 40 fresh rice seedlings were cut into 0.5 - to $1-\mathrm{m}$ strips with a razor blade and quickly transferred into $8-10 \mathrm{ml}$ of enzyme solution (1.5\% Cellulase R10, $0.75 \%$ Macerozyme R10, 0.6 M of mannitol, $10 \mathrm{mM}$ of MES, $10 \mathrm{mM}$ of $\mathrm{CaCl}_{2}$, and $0.1 \%$ bovine serum albumin (BSA), at $\mathrm{pH} 5.8$ ). Vacuum infiltration was applied for $30 \mathrm{~min}$ and, then strips were digested by shaking at $60-80 \mathrm{rpm}$ for $6-8 \mathrm{~h}$ at $25^{\circ} \mathrm{C}$ in the dark. The digested products were filtered with $40-\mu \mathrm{m}$ nylon mesh into a $50-\mathrm{ml}$ tube with $10 \mathrm{ml}$ of W5 buffer. The protoplasts were collected through centrifugation at $100 \times \mathrm{g}$ for $5 \mathrm{~min}$ and then resuspended with $10 \mathrm{ml}$ of W5 buffer. This step was repeated with centrifugation at $100 \times g$ for $2 \mathrm{~min}$ at room temperature. The cells were then suspended in MMG buffer $(0.4$ 
$\mathrm{M}$ of mannitol, $4 \mathrm{mM}$ of MES, and $15 \mathrm{mM}$ of $\mathrm{MgCl}_{2}$, at $\mathrm{pH}$ 5.8) for $2.6 \times 10^{-6}$ cells $/ \mathrm{ml}$. Two hundred microliters of protoplasts was mixed with $30 \mu \mathrm{l}$ of plasmid $(30 \mu \mathrm{g})$ and $230 \mu \mathrm{l}$ of PEG buffer (40\% w/v PEG4000, 0.2 M of mannitol, and 0.1 $\mathrm{M}$ of $\mathrm{CaCl}_{2}$ ) for an incubation of $20 \mathrm{~min}$ at room temperature. After $900 \mu \mathrm{l}$ of W5 buffer to stop transformation was added, the protoplasts were centrifuged at $250 \times g$ for $5 \mathrm{~min}$ and resuspended in $1 \mathrm{ml}$ of washing and incubation (WI) buffer ( $0.5 \mathrm{M}$ of mannitol, $20 \mathrm{mM}$ of $\mathrm{KCl}$, and $4 \mathrm{mM}$ of MES at $\mathrm{pH} 5.7$ ), before being transferred into three 6-well culture plate for $32^{\circ} \mathrm{C}$ incubation. After incubation for $48 \mathrm{~h}$, the protoplasts were collected for DNA extraction and further analysis. Each protoplast transformation experiment was performed in three biological replicates.

\section{Rice Stable Transformation}

Rice stable transformation was conducted as published previously with the Agrobacterium tumefaciens strain EHA105 (Zheng et al., 2016; Zhou et al., 2017; Zhou et al., 2019). The rice seeds were sterilized with $70 \%$ ethanol for $1 \mathrm{~min}$ and washed with sterile water, and then $2.5 \%$ sodium hypochlorite containing a drop of Tween 20 was added for 15 min of shaking. After being washed with sterile water, these seeds were sterilized in $2.5 \%$ sodium hypochlorite for another $15 \mathrm{~min}$. Afterwards, these seeds were washed with sterile water and cultured on solid medium at $28^{\circ} \mathrm{C}$ for $2-3$ weeks. Agrobacterium cultures were resuspended in liquid medium $(\mathrm{OD} 600=0.06-0.1)$ containing $100 \mu \mathrm{M}$ of acetosyringone. The fresh rice calli were chosen and immersed in the Agrobacterium liquid medium for $2 \mathrm{~min}$. These calli were collected and cultured in solid medium at $25^{\circ} \mathrm{C}$ in a dark growth chamber. After 3 days, these calli were washed with sterile water and transferred onto screening medium at $32^{\circ} \mathrm{C}$ with a $12-\mathrm{h}$ light/12-h dark photoperiod for 2 weeks. Then, growing calli were moved onto regenerative medium at $28^{\circ} \mathrm{C}$ with a $16-\mathrm{h}$ light/8-h dark cycle. After 3-4 weeks, the transgenic rice plants were grown into seedlings for subsequent analyses.

\section{Mutation Analysis by CAPS}

Genomic DNA was extracted from transformed rice protoplasts or transgenic rice using the cetyl trimethylammonium bromide (CTAB) method (Stewart and Via, 1993). Mutagenesis at target sites was analyzed by cleaved amplified polymorphic sequence (CAPS) with corresponding enzymes. The OsPDS-sgRNA01 site was amplified with primers OsPDS-F1 and OsPDS-R1, and the polymerase chain reaction (PCR) product was digested with the enzyme PstI (Table S1). The OsDEP1-sgRNA01 site was amplified with primers OsDEP1-F and OsDEP1-R (Table S1) and then digested with the enzyme $M f e I$. All digested products were resolved on $1 \%$ agarose gels.

\section{High-Throughput Sequencing Analysis}

High-throughput sequencing analysis was carried out as published previously (You et al., 2018). Genome regions of targeted sites were PCR amplified using high-throughput primers (Table S1). The OsPDS-sgRNA01 site was amplified with primers Cas9-OsPDS-HTS-F1 and Cas9-OsPDS-HTS-R1. The OsDEP1sgRNA01 site was amplified with primers Cas9-OsDEP1-HTS-F1 and Cas9-OsDEP1-HTS-R1. The PCR products were purified by
Qubit 2.0 Fluorometer (Life Technologies) and then sequenced using Illumina HiSeq 2500 platform. Each sample generated more than 50,000 reads. Raw sequencing data were analyzed by CRISPRMatch (You et al., 2018). The mean averages and standard deviations of three biologically independent replicates were calculated. To compare two conditions, the two-tailed test was used, assuming unequal variance between samples.

\section{RESULTS}

\section{Genome Editing in Rice Cells With an Engineered BiP CRISPR-Cas9 System}

To demonstrate that we can expression CRISPR-Cas9 with a $\mathrm{BiP}$, we first engineered a $\mathrm{BiP}$ based on CaMV 35s minimal promoter (Xie et al., 2001). As illustrated in Figure 1A, two CaMV 35s minimal promoters flanking an Arabidopsis enhancer sequence (Zhu et al., 2015; Zhang et al., 2016) were positioned in opposite directions, with one driving Cas9 expression and the other one driving the sgRNA. As a negative control, a minimal BiP without the Arabidopsis enhancer sequence was used. A BiP, unlike Pol III promoters such as U6 or U3, utilized RNA polymerase II for transcription. We used a Csy4 RNase system for precise processing of the sgRNA (Tsai et al., 2014; Cermak et al., 2017), so that it will not have a $5^{\prime}$ cap and $3^{\prime}$ poly A sequence. We designed one sgRNA each for targeting $O s P D S$ and $O s D E P 1$ in rice. The resulting T-DNA constructs were used to transform rice protoplasts. Targeted mutagenesis was assessed by CAPS analysis, where uncut bands indicate targeted mutations induced by Cas9. The results show that the mini 35s-enhancer-Csy4 $\mathrm{BiP}$ system resulted in detectable mutations, which were absent from the mini 35s-Csy4 negative control samples (Figures 1B, C). Deep sequencing of PCR amplicons was used to further quantify the mutation frequency at each target site. Consistent with the CAPS result, mutation frequencies of $\sim 10 \%$ and $\sim 12 \%$ were found for OsPDS and OsDEP1 target sites, respectively (Figures 1D, E).

\section{Targeted Mutagenesis in Rice TO Lines With the Mini 35s-Enhancer BiP CRISPR- Cas9 System}

To further assess the mini 35S-enhancer-Csy4 BiP system, we used the two working T-DNA constructs to generate stable transgenic rice lines. For the OsPDS target site, we screened 29 T0 lines using CAPS with restriction digestion by Pst (Figure 2A). For the OsDEP1 target site, we screened 34 T0 lines using CAPS with restriction digestion by $\mathrm{MfeI}$ (Figure 2B). Mutant lines with uncut bands were subjected for Sanger sequencing and decoding for genotype. Among 29 T0 lines in which OsPDS was targeted, six lines carried mutations (20.69\%), in which four were biallelic and two were heterozygous (Figure 2C and additional data: Figure S1). Among 34 T0 lines in which OsDEP1 was targeted, 18 lines carried mutations (52.94\%) in which seven were biallelic and the remainder were heterozygous (Figure 2C). The genome editing frequencies at both target sites appeared to be lower than those obtained with the conventional mixed dual 
A -OCS-T sgRNA mini 35S enhancer mini 35S NLS-csy4-P2A-Cas9-NLS Hsp-Tcsy4 recognition site
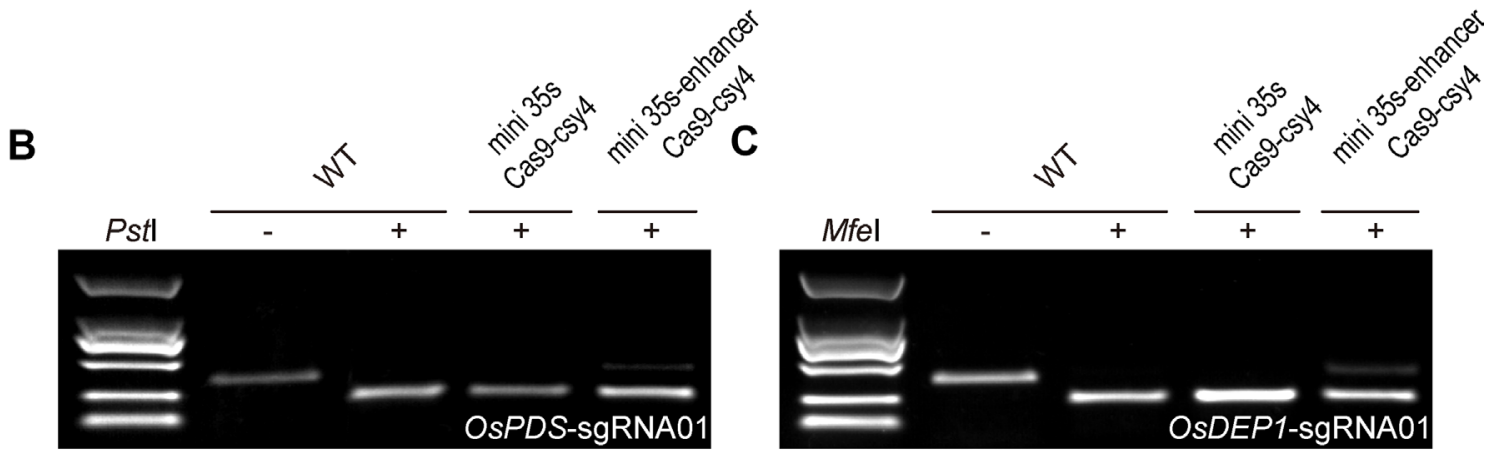

D

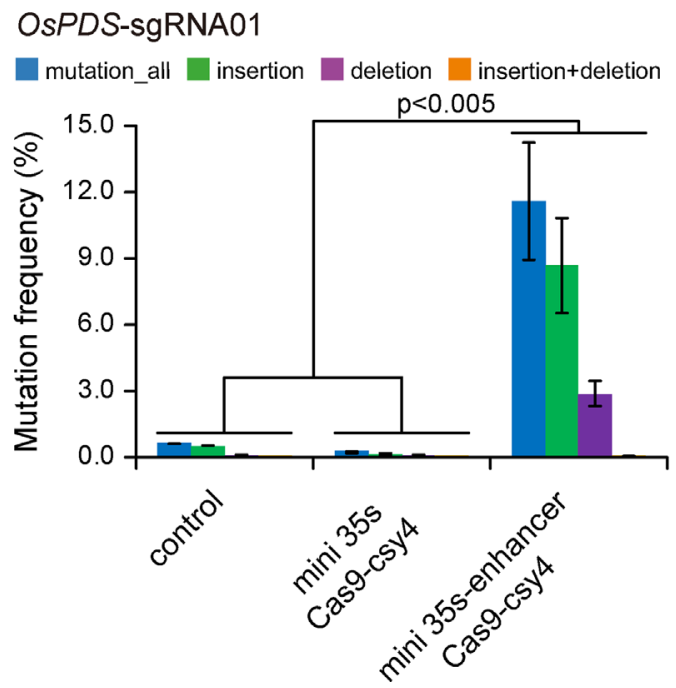

E

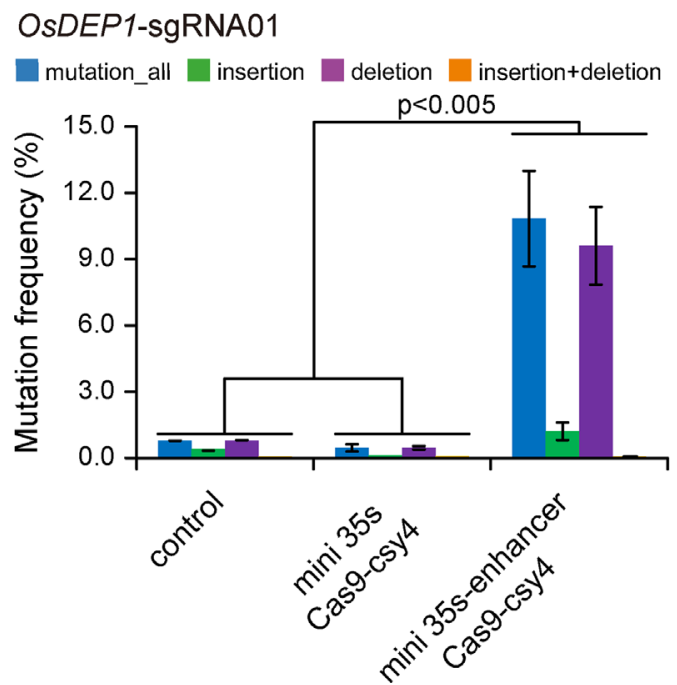

FIGURE 1 | Expression of CRISPR-Cas9 with an engineered bidirectional promoter system (A) Diagrams of expression cassettes. (B) Cleaved amplified polymorphic sequence (CAPS) analysis of mutation frequencies at the OsPDS target site. (C) CAPS analysis of mutation frequencies at the OsDEP1 target site. (D) Quantification of mutagenesis at the OsPDS target site by deep sequencing. (E) Quantification of mutagenesis at the OsDEP1 target site by deep sequencing. Bar graphs show average mutation frequency from three biologically independent replicates with error bars representing standard deviations $(n=3)$. The statistical analyses were performed using the two-tailed test.

promoter CRISPR-Cas9 system (Lowder et al., 2015) or with the STU CRISPR-Cas9 system (Tang et al., 2016; Tang et al., 2019), indicating room for improvement.

\section{Improved Marker Gene Expression by a Rice Endogenous BiP System}

While we recognized that stronger BiP systems could be engineered by using stronger enhancers or multiple copies of enhancers, we reasoned that utilization of a plant endogenous $\mathrm{BiP}$ system may represent a straightforward strategy for improvement. Based on RNAseq and cDNA microarray data analysis, a recent study identified a constitutive $\mathrm{BiP}$ of high expression in rice, $\mathrm{OsBiP} 1$, which drives expression of Os02g42314 at the $5^{\prime}$ end and Os02g42320 at the $3^{\prime}$ end (Wang et al., 2016). We decided to test OsBiP1 in our study and compared its expression strength by positioning a GFP reporter gene at the $3^{\prime}$ end of this $\mathrm{BiP}$. We compared OsBiP1 with the engineered mini 35s-enhancer BiP, mini 35S BiP (negative control), and $\mathrm{ZmUbi}$, which is a strong unidirectional promoter commonly used for expression of Cas genes in rice and other monocots (Tang et al., 2017; Lee et al., 2019) (Figure 3A). We transiently transformed the four GFP constructs in rice protoplasts and measured GFP signals among total cells (Figure 3B). Based on the quantification of GFP-positive cells (Figure 3B) and GFP intensity in positive cells (Figure 3C), OsBiP1 displayed stronger expression than mini 35s-enhancer and mini 35s BiPs yet weaker expression than the ZmUbi promoter. Nevertheless, the results suggest that the use of plant endogenous BiPs may improve expression over the engineered mini 35s-enhancer system. 
A

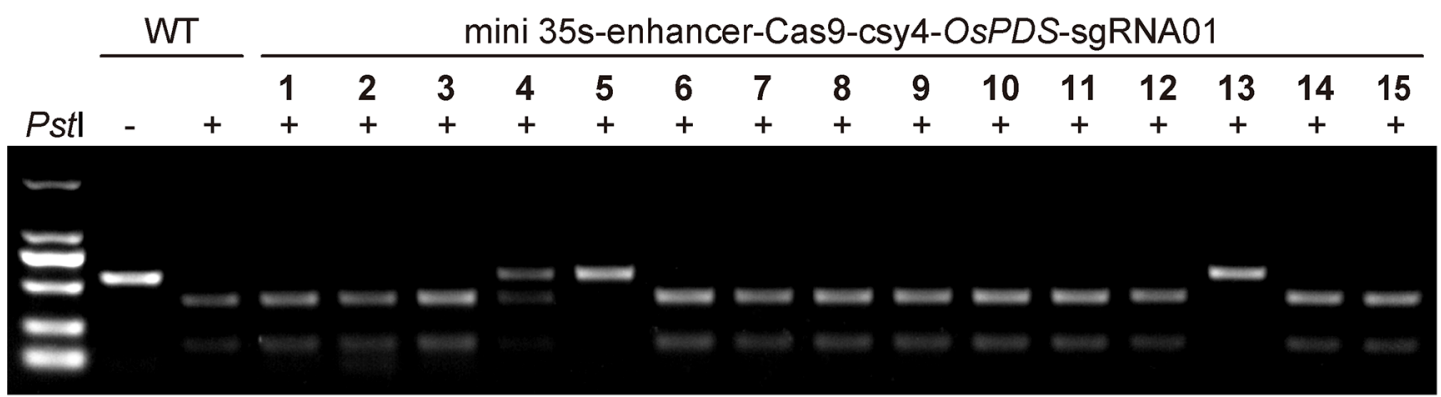

B

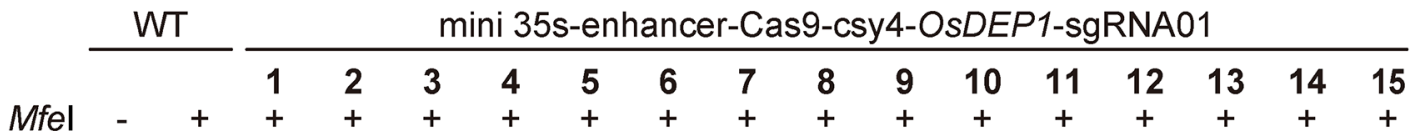

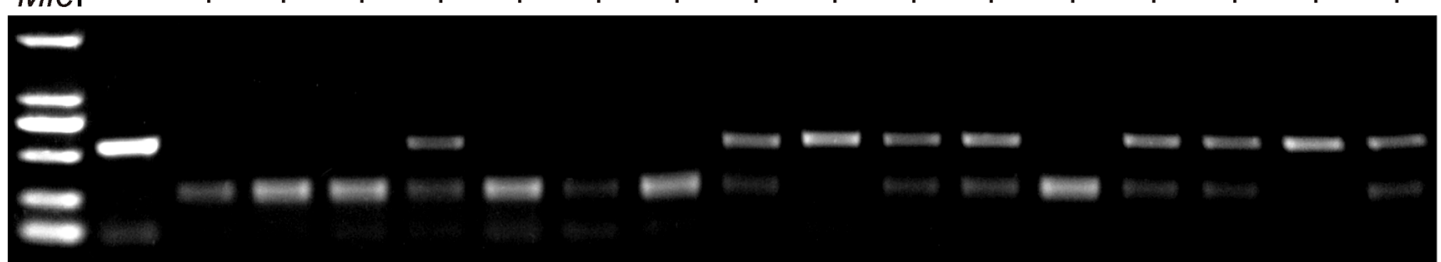

C

\begin{tabular}{ccccc}
\hline $\begin{array}{c}\text { Targeted } \\
\text { rice sites }\end{array}$ & System & $\begin{array}{c}\text { Tested } \\
\text { T0 lines }\end{array}$ & $\begin{array}{c}\text { Mutated T0 lines } \\
\text { (number; ratio) }\end{array}$ & $\begin{array}{c}\text { Biallelic mutation lines } \\
\text { (number; ratio) }\end{array}$ \\
\hline OsPDS-sgRNA01 & enhancer-csy4 & 29 & $6 ; 20.7 \%$ & $4 ; 66.7 \%$ \\
OsDEP1-sgRNA01 & enhancer-csy4 & 34 & $18 ; 52.9 \%$ & $7 ; 38.9 \%$ \\
\hline
\end{tabular}

FIGURE 2 | Generation of rice T0 mutants with CRISPR-Cas9 expressed by an engineered bidirectional promoter (A) Cleaved amplified polymorphic sequence (CAPS) analysis for targeted mutations at OSPDS among T0 lines. Note that only results of the first 15 lines were shown. (B) CAPS analysis for targeted mutations at OsDEP1 among TO lines. Note that only results of the first 15 lines were shown. (C) Summary of genotyping results of all T0 lines analyzed.

\section{The OsBip1 BiP System Results in Improved CRISPR-Cas9 Genome Editing in Rice Cells}

We then tested OsBiP1 for the expression of CRISPR-Cas9. In our design, the sgRNA was put at the $5^{\prime}$ end of OsBiP1 and Cas9 was put at the $3^{\prime}$ end. We also sought to compare the Csy4 system with tRNA, which is another efficient polycistronic sgRNA processing system (Xie et al., 2015) (Figure 4A). Both OsBiP1-Cas9-tRNA and OsBiP1-Cas9-Csy4 systems were used to target the same two target sites at OsPDS and OsDEP1 in rice protoplasts. In both cases, comparable mutation frequencies were detected at either target site with both systems (Figures 4B, C). Deep sequencing of PCR amplicons revealed mutation frequency of $\sim 16 \%$ at OsPDS and $\sim 40 \%$ at OsDEP1 (Figures 4D, E). The mutation frequencies by OsBiP1 were significantly higher ( $t$-test, $p<0.005)$ than those by the engineered mini 35s-enhancer BiP system (Figures 1B-E). At the OsPDS target site, there were more insertions than deletions (Figure 4D). At the OsDEP1 target site, there were more deletions than insertions (Figure 4E). These data were consistent with earlier observations (Figures 1D, E). We then looked into deletion profiles regarding deletion positions.
Consistent with mutation frequencies, the deletion profiles were largely similar for tRNA and Csy4 at the OsPDS target site and $O s D E P 1$ target site (Figure 4F).

\section{High-Efficiency Genome Editing in Rice T0 Lines With the OsBiP1 BiP CRISPR- Cas9 System}

To test whether the OsBiP1 CRISPR-Cas9 system could lead to high-frequency targeted mutagenesis in stable transgenic rice lines, we generated many T0 plants for each of the four T-DNA constructs targeting OsPDS and OsDEP1. CAPS analysis revealed high frequency of uncut bands in these T0 lines with OsBiP1Cas9-tRNA and OsBiP1-Cas9-Csy4 targeting OsPDS (Figures 5A, B), suggesting biallelic or homozygous mutations. Indeed, loss-offunction albino phenotype was observed for such lines (Figure 5C). The genotypes of these mutants were confirmed with Sanger sequencing. Interestingly, most T0 mutant lines carried 1-bp insertion/deletion (indel) mutations (Figures 5D, E). T0 lines with OsBiP1-Cas9-tRNA and OsBiP1-Cas9-Csy4 targeting OsDEP1 were also genotyped by CAPS and Sanger sequencing (additional data: Figures S2 and S3). At OsPDS, OsBiP1-Cas9-tRNA and OsBiP1-Cas9-Csy4 resulted in mutation frequencies of $77.1 \%$ and 
A

mini 35s-GFP:

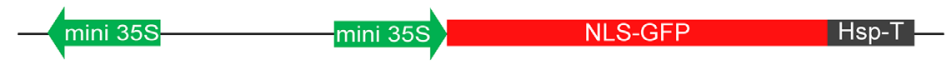

mini 35s-enhancer-GFP:

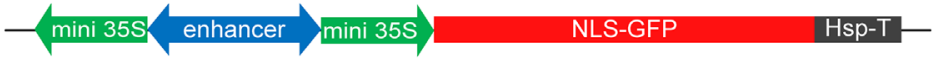

OsBiP1-GFP:

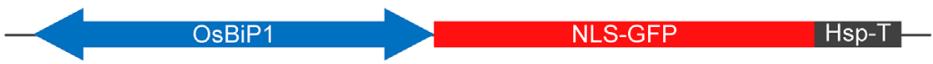

ZmUbi-GFP:

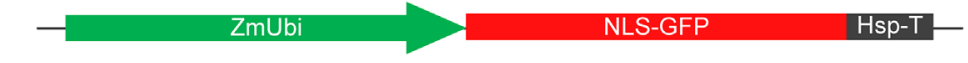

B

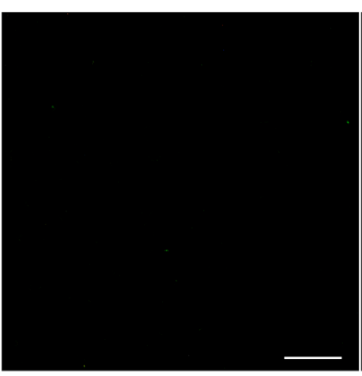

mini 35s-GFP

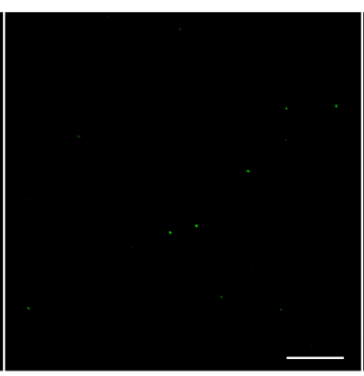

mini 35s-enhancer-GFP

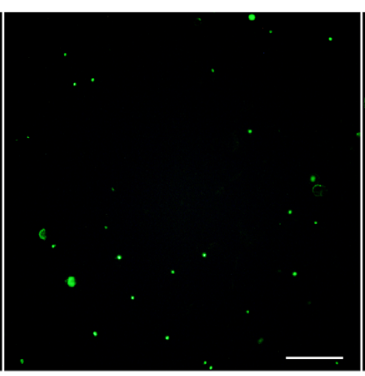

OsBiP1-GFP
C
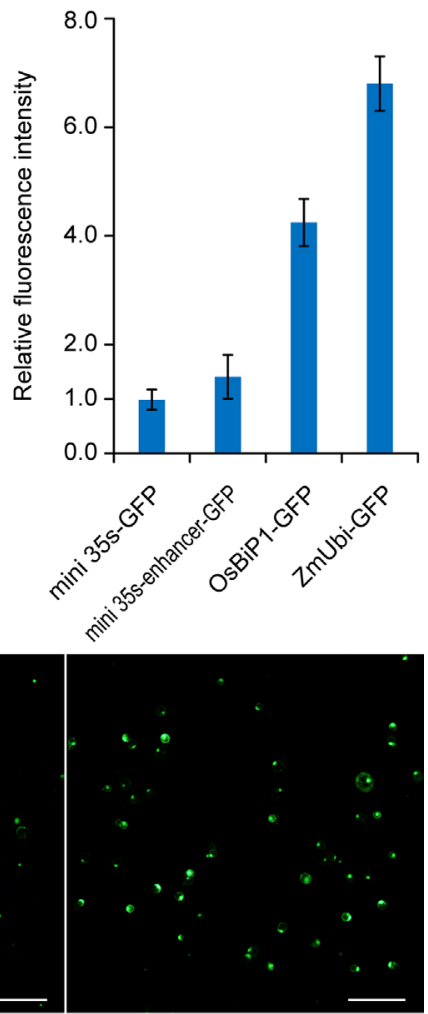

ZmUbi-GFP

FIGURE 3 | Comparison of different promoter systems in rice protoplasts (A) Diagrams of reporter constructs driven by different promoters. (B) Representative images of green-fluorescent protein (GFP)-expressing protoplasts among different treatments. (C) Quantification of relative fluorescence intensity for GFP-positive cells among different treatments. Bar graphs show average mutation frequency from three biologically independent replicates with error bars representing standard deviations $(n=3)$.

75.9\%, respectively (Figure 5F). Among them, biallelic mutation frequencies were $37.1 \%$ and $54.5 \%$. At OsDEP1, OsBiP1-Cas9tRNA and OsBiP1-Cas9-Csy4 generated mutation frequencies of $86.6 \%$ and $93.3 \%$, respectively (Figure 5F). Among them, biallelic mutation frequencies were $69.2 \%$ and $82.1 \%$. These results suggest significant improvement over the engineered mini 35s-enhancer BiP system. The OsBiP1-based CRISPR-Cas9 system appeared to have similar genome editing efficiency to our previously established mixed dual promoter or STU systems (Lowder et al., 2015; Tang et al., 2016; Tang et al., 2019).

\section{DISCUSSION}

Conventionally, CRISPR-Cas9 is expressed by a mixed dual promoter strategy in which Cas9 is expressed by a Pol II promoter and the sgRNA is expressed by a Pol III promoter such as U6 or U3. Most studies in plants have applied this mixed dual promoter systems as demonstrated in those initial studies showcasing CRISPR-Cas9 functionality in plants (Jiang et al., 2013; Li et al., 2013; Nekrasov et al., 2013; Fauser et al., 2014). Under this strategy, multiple sgRNAs can be expressed by stacking sgRNA expression cassettes (Li et al., 2013; Lowder et al., 2015), by using a tRNA processing system (Xie et al., 2015; Tang et al., 2019), by using hammerhead ribozyme
(HH)-hepatitis delta virus ribozyme (HDV) (Gao and Zhao, 2014; He et al., 2017; Tang et al., 2019), or by using the Csy4 RNase system (Cermak et al., 2017; Tang et al., 2019). While it is convenient to use Pol III promoters for sgRNA expression, these promoters generate shorter transcripts and tend to have weaker expression strength than do some Pol II promoters. Additionally, the use of Pol II promoters for sgRNA expression would allow for better spatiotemporal control when needed. For coordinated expression, it is more desirable to have Cas9 and the sgRNA under the same or similar promoters. Hence, it is very valuable to develop CRISPR-Cas9 expression systems in which sgRNAs are also expressed by Pol II promoters.

There are generally three strategies to express guide RNAs by Pol II promoters. The first strategy is a STU strategy that Cas 9 and sgRNA(s) are expressed by a single Pol II promoter as a single transcript. This strategy has been demonstrated for single or multiplexed genome editing in plants with sgRNAs being processed by HH ribozyme (Tang et al., 2016; Tang et al., 2019), tRNA (Tang et al., 2019), Csy4 (Tang et al., 2019), or plant endogenous RNase activities (Mikami et al., 2017). The STU strategies represent the most compact CRISPR expression systems. Given Cas and the sgRNA are expressed from the same transcript, it leaves little room for fine-tuning both components for optimal production of the Cas-guide RNA RNP complex. The second strategy is a dual Pol 
A

OsBiP1-Cas9-tRNA:

-OCS-T $\underset{\substack{\text { N SgRNA } \\ \text { tRNA coding site }}}{\text { OsBiP1 }}$

OsBip1-Cas9-csy4:

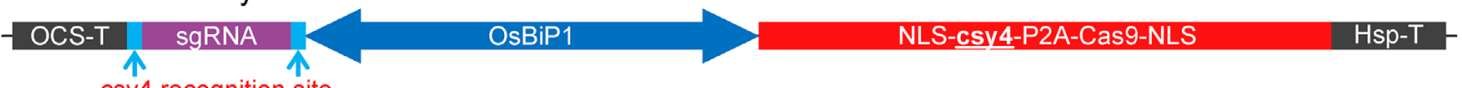

csy4 recognition site

B

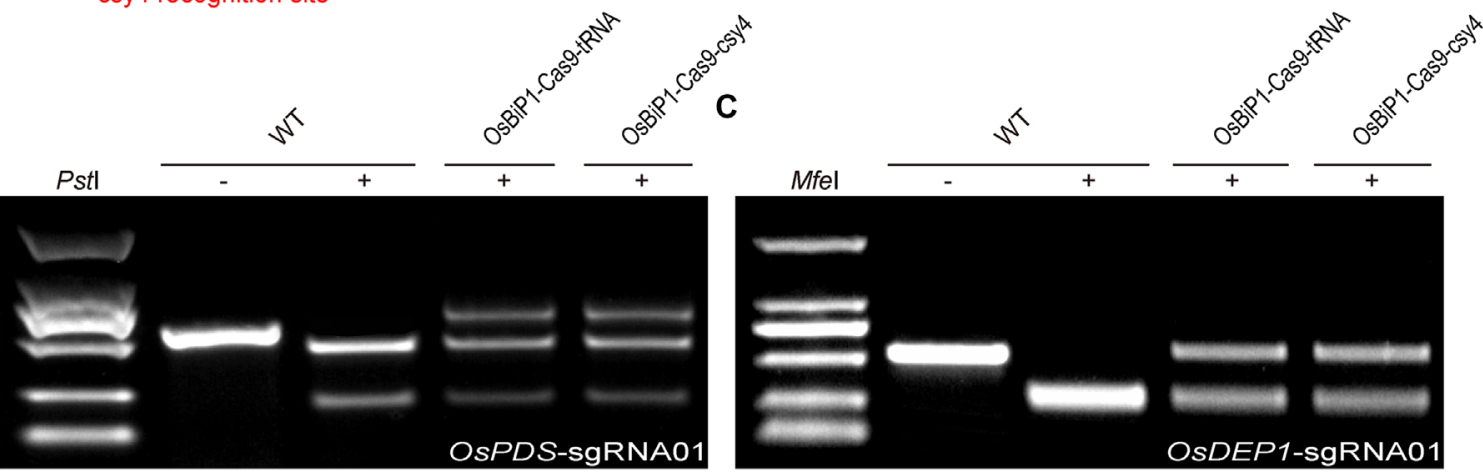

D

OsPDS-sgRNA01

mutation_all insertion deletion insertion+deletion
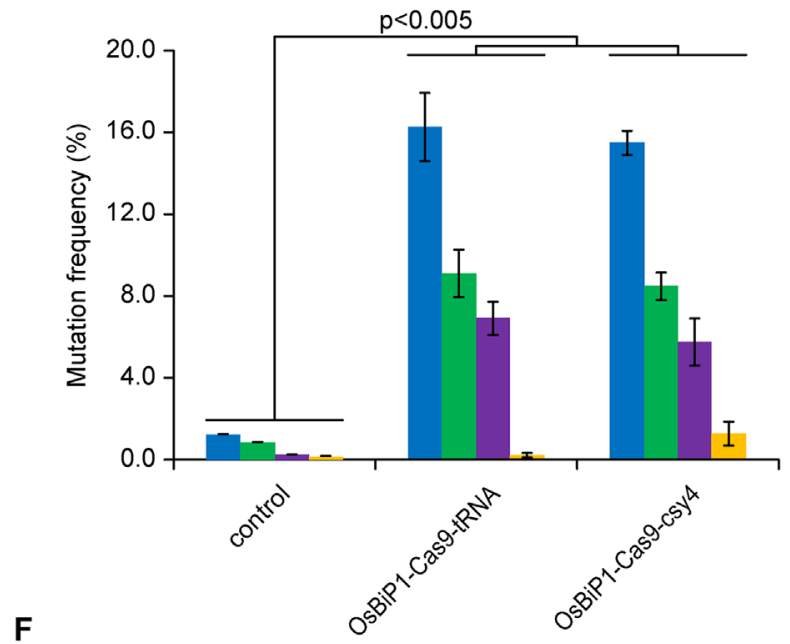

$\mathbf{F}$

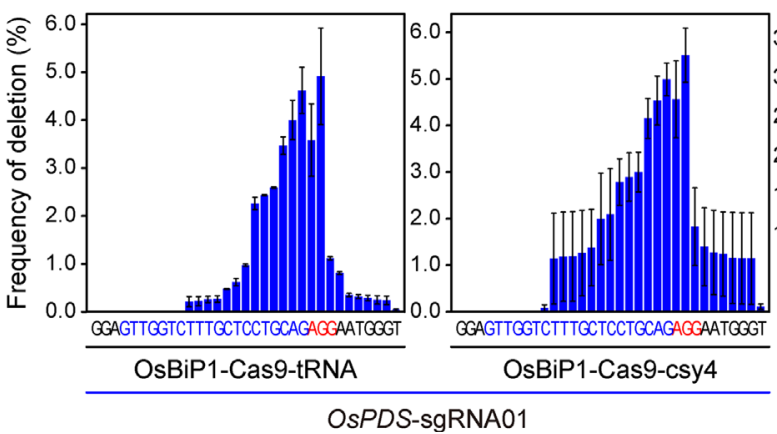

E

OsDEP1-sgRNA01

mutation_all insertion deletion insertion+deletion
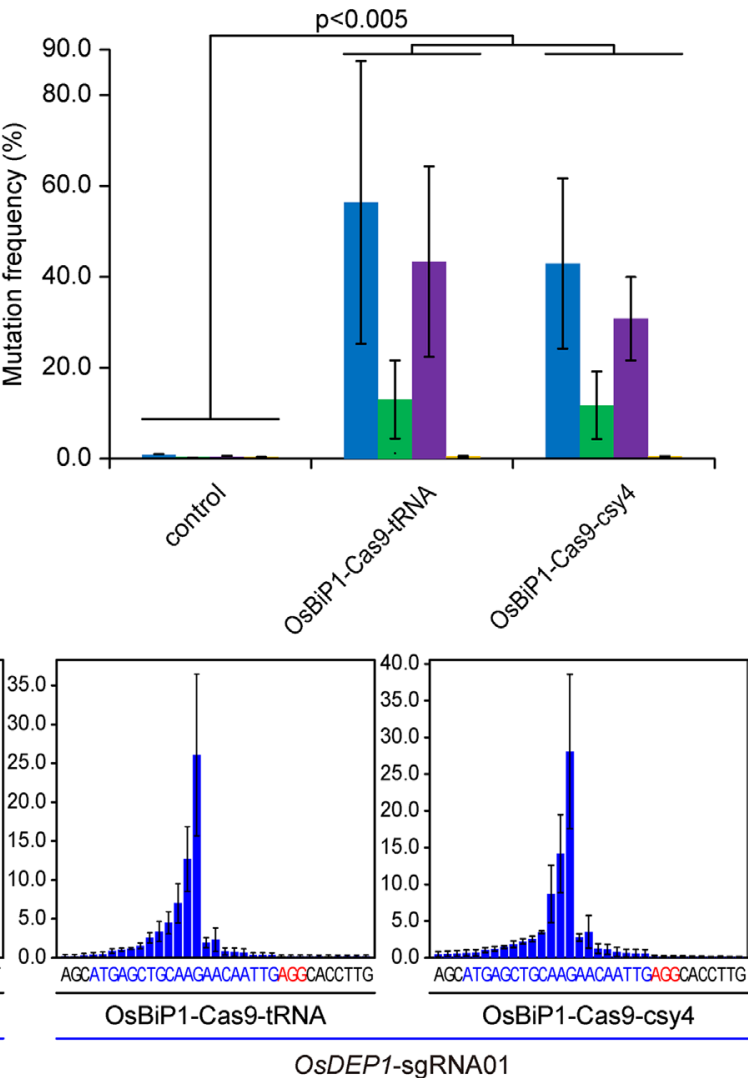

FIGURE 4 | Improved editing efficiency with the OsBiP1 bidirectional promoter (A) Diagrams of bidirectional promoter (BiP) CRISPR-Cas9 expression constructs in which single guide RNAs (sgRNAs) are processed by tRNA and Csy4. (B) Cleaved amplified polymorphic sequence (CAPS) analysis of mutagenesis efficiency at OsPDS. (C) CAPS analysis of mutagenesis efficiency at OsDEP1. (D) Quantification of mutagenesis at the OsPDS target site by deep sequencing. (E) Quantification of mutagenesis at the OsDEP1 target site by deep sequencing. (F) Mutation profile on deletion positions at the OsPDS and OsDEP1 target sites by OsBiP1-Cas9tRNA and OsBiP1-Cas9-Csy4. Bar graphs show average mutation frequency from three biologically independent replicates with error bars representing standard deviations $(n=3)$. The statistical analyses were performed using the two-tailed test. 
A

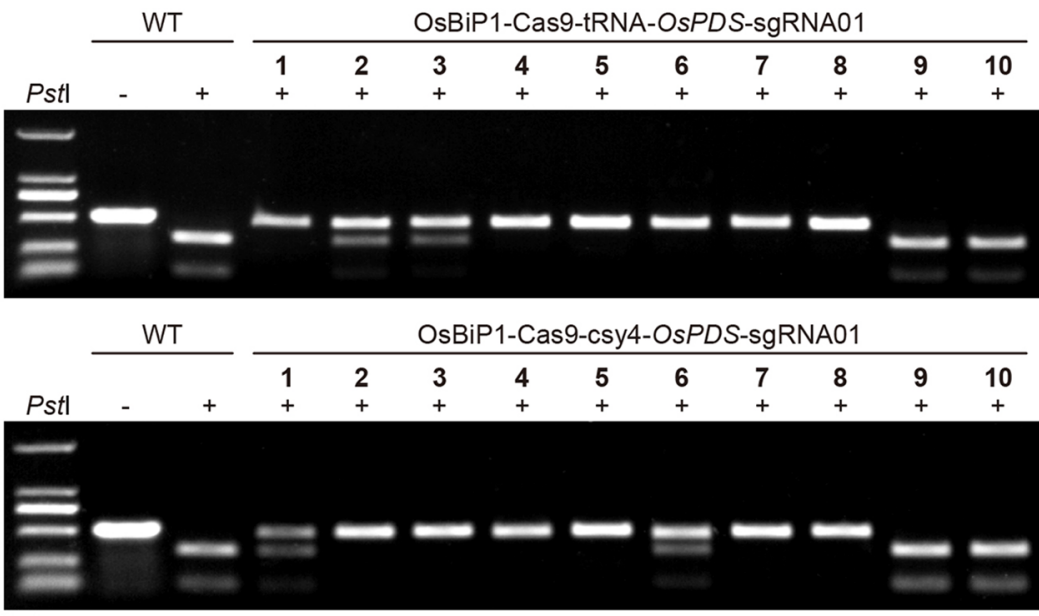

D

OsBiP1-Cas9-tRNA: : OsPDS-sgRNA01

OSPDS_WT GAGTTGGTCTTTGCTCCTG-CAGAGGAA

\# 01 :

allele1:

allele2:

\#02:

allele1:

allele2:

\#03:

allele1:

allele2:

\#04:

allelel:

allele2:

\#05:

allelel:

allele2:

\#06:

allele1:

allele2:

\#07:

allelel:

allele2:

\#08:

allele1:

allele2:
GAGTTGGTCTTTGCTCCTGCCAGAGGAA +1bp GAGTTGGTCTTTGCTCCTGgCAGAGGAA +1bp

GAGTTGGTCTTTGCTCCTG-CAGAGGAA WT GAGTTGGTCTTTGCTCCTGaCAGAGGAA +1bp

GAGTTGGTCTTTGCTCCTG-CAGAGGAA WT GA

GAGTTGGTCTTTGCTCCTGtCAGAGGAA +1bp GAGTTGGTCTTTGCTCCTGECAGAGGAA +1bp

GAGTTGGTCTTTGCTCCTGCCAGAGGAA +1bp GAGTTGGTCTTTGCTCCTGACAGAGGAA +1bp

GAGTTGGTCTTTGCTCCTGECAGAGGAA +1bp GAGTTGGTCTTTGCTCCTGaCAGAGGAA +1bp

GAGTTGGTCTTTGCTCCTGLCAGAGGAA +1bp GAGTTGGTCTTTGCTCCTGECAGAGGAA +1bp

GAGTTGGTCTTTGCTCCTGECAGAGGAA +1bp GA
$\mathbf{E}$

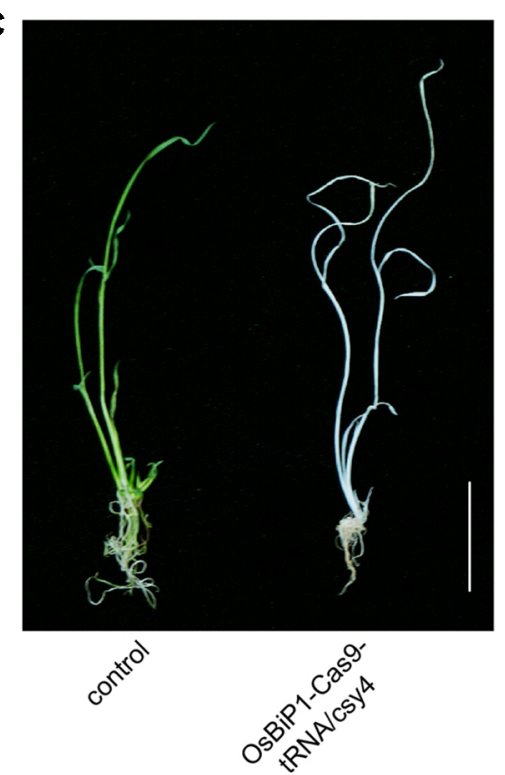

OsBiP1-Cas9-Csy4::OsPDS-sgRNA01

OSPDS_WT GAGTTGGTCTTTGCTCCTG-CAGAGGAA

\#01:

allele1: GAGTTGGTCTTTGCTCCTGCCAGAGGAA +1bp

allele2: GAGTTGGTCTTTGCTCCTG-CAGAGGAA WT

\#02:

allelel: GAGTTGGTTTTGCTCCT--CAGAGGA -1bp

allele2: GA

\#03:

allele1: GAGTTGGTCTTTGCTCCTgtCAGAgGA +1bp

allele2: GAGTTGGTCTTTGCTCCTGaCAGAGGAA +1bp

\#04:

allelel: GAGTTGGTCTTTGCTCCTGgCAGAGGAA +1bp

allele2: GA

\#05:

allele1: GAGTTGGTCTTTGCTCCTGaCAGAGGAA +1bp

allele2: GAGTTGGTCTTTGCTCCTGaCAGAGGAA +1bp

\#06:

allele1: GAGTTGGTCTTTGCTCCTG-CAGAGGAA WT

allele2: GA

\#07:

allele1: GAGTTGGTCTTTGCTCCTGtCAGAGGAA +1bp

allele2: GAGTTGGTCTTTGCTCCTGtCAGAGGAA +1bp

\#08:

allele1: GAGTTGGTCTTTGCTCCTGCCAGAGGA +1bp allele2: $\quad$ GAGTTGGTCTTGCTCCTGCCAGAGGAA +1bp

$\mathbf{F}$

\begin{tabular}{ccccc}
\hline $\begin{array}{c}\text { Targeted } \\
\text { rice sites }\end{array}$ & System & $\begin{array}{c}\text { Tested } \\
\text { T0 lines }\end{array}$ & $\begin{array}{c}\text { Mutated T0 lines } \\
\text { (number; ratio) }\end{array}$ & $\begin{array}{c}\text { Biallelic mutation lines } \\
\text { (number; ratio) }\end{array}$ \\
\hline OsPDS-sgRNA01 & OsBiP1-tRNA & 42 & $35 ; 77.1 \%$ & $13 ; 37.1 \%$ \\
OsPDS-sgRNA01 & OsBiP1-csy4 & 29 & $22 ; 75.9 \%$ & $12 ; 54.5 \%$ \\
\hline OsDEP1-sgRNA01 & OsBiP1-tRNA & 30 & $26 ; 86.6 \%$ & $18 ; 69.2 \%$ \\
OsDEP1-sgRNA01 & OsBiP1-csy4 & 30 & $28 ; 93.3 \%$ & $23 ; 82.1 \%$ \\
\hline
\end{tabular}

FIGURE 5 | High-efficiency generation of rice T0 mutants with the OsBiP1-based CRISPR-Cas9 system (A) Cleaved amplified polymorphic sequence (CAPS) analysis for targeted mutagenesis at OsPDS among 10 TO lines. (B) CAPS analysis for targeted mutagenesis at OsDEP1 among 10 T0 lines. (C) A representative photo showing the wild-type control (left) and an OSPDS biallelic knockout plant (right). (D) Genotyping results of T0 lines with OsPDS targeted by OsBiP1-Cas9tRNA. Note that only results from eight out of 42 TO lines are shown. (E) Genotyping results of T0 lines OsPDS targeted by OsBiP1-Cas9-Csy4. Note that only results from 8 out of 29 TO lines are shown. Indel events are highlighted in red in lowercase. The PAM is highlighted in red in uppercase. (F) Summary of genotyping results for all TO lines targeted by OsBiP1-Cas9-tRNA and OsBiP1-Cas99-Csy4 at OsPDS and OsDEP1, respectively. 
II promoter strategy where Cas9 and the sgRNA are expressed by separate Pol II expression units. For example, high-efficiency genome editing was achieved by using a strong Pol II promoter (CmYLCV) for expressing sgRNAs, and the editing frequencies were almost twice as much as those obtained with a Pol III promoter (AtU6) (Cermak et al., 2017). Consistent with this result, a dual Pol II promoter system also contributed to highly efficient genome editing by CRISPR-Cas12a (Tang et al., 2017; Zhong et al., 2018; Tang et al., 2019). The third strategy is a BiP system that uses a single Pol II promoter to drive Cas9 and sgRNAs in opposite directions, an idea explored in our study here. An artificial or engineered $\mathrm{BiP}$ system was previously demonstrated in plants (Xie et al., 2001) and was recently applied for coordinated multi-gene expression in maize for developing gene stacked GM plants (Kumar et al., 2015). It is also very common to find endogenous BiP systems in plants, such as the $\mathrm{BiP}$ that drives expression of $\mathrm{Cab} 1$ and $\mathrm{Cab} 2$ in Arabidopsis (Mitra et al., 2009). In this study, we showed that both engineered BiP and plant endogenous $\mathrm{BiP}$ systems can be used for expression CRISPRCas for efficient genome editing.

Among four possible CRISPR-Cas9 expression strategies (e.g., mixed dual promoters, dual Pol II promoters, STU, and $\mathrm{BiP})$, a BiP system balances expression strength, compactness, and fine-tunability. In this proof-of-concept study, the rice endogenous OsBiP1 system is more efficient than the engineered BiP system based on the mini $35 \mathrm{~s}$ promoter. We, however, want to point out that this may not be always the case because both $\mathrm{BiP}$ strategies, whether engineered or endogenous, can be further improved. First, rational designbased approach could be used to engineer BiP systems with improved expression strength and stability in plants, as was done in Escherichia coli and yeast (Yang et al., 2013; Elison et al., 2018). Second, aided by genomic and transcriptome data sets, many endogenous BiP systems could be identified and tested, as was done in rice (Wang et al., 2016). Third, different $3^{\prime}$-UTR and terminator sequences could be tested for tuning the expression of Cas9 and guide RNAs as well as tissue specificity (Lianoglou et al., 2013). Finally, even though we only worked with CRISPR-Cas9 in this study, it is conceivable that $\mathrm{BiP}$ strategies are readily applicable for the expression of CRISPR-Cas12a for genome editing and CRISPR-Cas13 for transcriptome editing (Abudayyeh et al., 2017). All these exiting fronts are awaiting future exploration.

\section{DATA AVAILABILITY}

All datasets generated for this study are included in the manuscript and the Supplementary Files.

\section{AUTHOR CONTRIBUTIONS}

$\mathrm{YZ}$ conceived the project. $\mathrm{YZ}, \mathrm{YQ}$, and $\mathrm{TZ}$ designed the experiments. QR and XT generated all constructs. QR and ZZ performed the transient assays in protoplasts. QR, YW, QL, MY,
$\mathrm{YH}, \mathrm{CQ}$, and $\mathrm{XZ}$ generated stable transgenic rice and identified the rice mutants. QY and TZ conducted the next-generation sequencing (NGS) data analysis. YZ, YQ, QR, and ZZ analyzed the data and wrote the manuscript draft. All authors read and approved the final manuscript.

\section{ACKNOWLEDGMENTS}

This work was supported by the Sichuan Youth Science and Technology Foundation (2017JQ0005), the National Science Foundation of China (31771486), the Program for International Science and Technology Cooperation and Exchanges of Sichuan Province (2018HH0112), and the Science Strength Promotion Program of UESTC to YZ; the National Transgenic Major Project (2018ZX08020-003) and the Open Foundation of Jiangsu Key Laboratory of Crop Genomics and Molecular Breeding (PL201801) to YZ and TZ; the National Science Foundation of China (81872957) to XZ; the Jiangsu Specially-Appointed Professor and the Priority Academic Program Development of Jiangsu Higher Education Institutions (PAPD) to TZ; and the National Science Foundation Plant Genome Research Program (IOS1758745), USDA-NIFA Biotechnology Risk Assessment Research Program (2018-33522-28789), Foundation for Food and Agriculture Research (593603), and a Syngenta Biotechnology Research Grant to YQ. We thank Aimee A. Malzahn for proofreading this manuscript.

\section{SUPPLEMENTARY MATERIAL}

The Supplementary Material for this article can be found online at: https://www.frontiersin.org/articles/10.3389/fpls.2019.01173/ full\#supplementary-material

FIGURE S1 | TO rice plants targeted by OsPDS-sgRNA01 with the mini 35s $\neg$ enhancer-Cas9-Csy4 system. Sanger sequencing results for six TO lines. The target sequence is shown in blue. Indel events are highlighted in red in lower case. The PAM is highlighted in red in upper case.

FIGURE S2 | TO rice plants targeted by OSDEP1-sgRNA01 with the OsBiP1هCas9-tRNA system. (A) The OsBiP1-Cas9-tRNA system indel events were first identified by CAPS analysis. (B) Sanger sequencing results for TO lines. The target sequence is shown in blue. Indel events are highlighted in red in lower case. The PAM is highlighted in red in upper case.

FIGURE S3 | TO rice plants targeted by OsDEP1-sgRNA01 with the OsBiP1冈Cas9-Csy4 system. (A) The OsBiP1-Cas9-Csy4 system indel events were first identified by CAPS analysis. (B) Sanger sequencing results for TO lines. The target sequence is shown in blue. Indel events are highlighted in red in lower case. The PAM is highlighted in red in upper case.

FIGURE S4 | Sequence of an Arabidopsis enhancer used in this study.

FIGURE S5 | The OsBiP1 bidirectional promoter sequence used in this study. 


\section{REFERENCES}

Abudayyeh, O. O., Gootenberg, J. S., Essletzbichler, P., Han, S., Joung, J., Belanto, J. J., et al. (2017). RNA targeting with CRISPR-Cas13. Nature 550, 280-284. doi: 10.1038 /nature24049

Andersson, M., Turesson, H., Olsson, N., Falt, A. S., Ohlsson, P., Gonzalez, M. N., et al. (2018). Genome editing in potato via CRISPR-Cas9 ribonucleoprotein delivery. Physiol. Plant 164, 378-384. doi: 10.1111/ ppl.12731

Begemann, M. B., Gray, B. N., January, E., Gordon, G. C., He, Y., Liu, H., et al. (2017). Precise insertion and guided editing of higher plant genomes using Cpf1 CRISPR nucleases. Sci Rep. 7, 11606. doi: 10.1038/s41598-017-11760-6

Cermak, T., Curtin, S. J., Gil-Humanes, J., Cegan, R., Kono, T. J. Y., Konecna, E., et al. (2017). A multipurpose toolkit to enable advanced genome engineering in plants. Plant Cell 29, 1196-1217. doi: 10.1105/tpc.16.00922

Elison, G. L., Xue, Y., Song, R., and Acar, M. (2018). Insights into bidirectional gene expression control using the canonical GAL1/GAL10 promoter. Cell Rep. 25, 737-748 e734. doi: 10.1016/j.celrep.2018.09.050

Endo, A., Masafumi, M., Kaya, H., and Toki, S. (2016). Efficient targeted mutagenesis of rice and tobacco genomes using Cpf1 from Francisella novicida. Sci. Rep. 6, 38169. doi: 10.1038/srep38169

Fauser, F., Schiml, S., and Puchta, H. (2014). Both CRISPR/Cas-based nucleases and nickases can be used efficiently for genome engineering in Arabidopsis thaliana. Plant J. 79, 348-359. doi: 10.1111/tpj.12554

Gao, Y., and Zhao, Y. (2014). Self-processing of ribozyme-flanked RNAs into guide RNAs in vitro and in vivo for CRISPR-mediated genome editing. J. Integr. Plant Biol. 56, 343-349. doi: 10.1111/jipb.12152

He, Y., Zhang, T., Yang, N., Xu, M., Yan, L., Wang, L., et al. (2017). Self-cleaving ribozymes enable the production of guide RNAs from unlimited choices of promoters for CRISPR/Cas9 mediated genome editing. J. Genet. Genom. 44, 469-472. doi: 10.1016/j.jgg.2017.08.003

Jiang, W., Zhou, H., Bi, H., Fromm, M., Yang, B., and Weeks, D. P. (2013). Demonstration of CRISPR/Cas9/sgRNA-mediated targeted gene modification in Arabidopsis, tobacco, sorghum and rice. Nucleic Acids Res. 41, e188. doi: 10.1093/ nar/gkt780

Kumar, S., AlAbed, D., Whitteck, J. T., Chen, W., Bennett, S., Asberry, A., et al. (2015). A combinatorial bidirectional and bicistronic approach for coordinated multi-gene expression in corn. Plant Mol. Biol. 87, 341-353. doi: 10.1007/ s11103-015-0281-6

Lee, K., Zhang, Y., Kleinstiver, B. P., Guo, J. A., Aryee, M. J., Miller, J., et al. (2019). Activities and specificities of CRISPR-Cas9 and Cas12a nucleases for targeted mutagenesis in maize. Plant Biotechnol. J. 17, 362-372. doi: 10.1111/pbi.12982

Li, J. F., Norville, J. E., Aach, J., McCormack, M., Zhang, D., Bush, J., et al. (2013). Multiplex and homologous recombination-mediated genome editing in Arabidopsis and Nicotiana benthamiana using guide RNA and Cas9. Nat. Biotechnol. 31, 688-691. doi: 10.1038/nbt.2654

Liang, Z., Chen, K., Li, T., Zhang, Y., Wang, Y., Zhao, Q., et al. (2017). Efficient DNA-free genome editing of bread wheat using CRISPR/Cas9 ribonucleoprotein complexes. Nat. Commun. 8, 14261. doi: 10.1038/ ncomms14261

Lianoglou, S., Garg, V., Yang, J. L., Leslie, C. S., and Mayr, C. (2013). Ubiquitously transcribed genes use alternative polyadenylation to achieve tissue-specific expression. Genes Dev. 27, 2380-2396. doi: 10.1101/gad.229328.113

Lowder, L. G., Zhang, D., Baltes, N. J., Paul, J. W., Tang, X., Zheng, X., et al. (2015). A CRISPR/Cas9 toolbox for multiplexed plant genome editing and transcriptional regulation. Plant Physiol. 169, 971-985. doi: 10.1104/ pp. 15.00636

Lowder, L. G., Zhou, J., Zhang, Y., Malzahn, A., Zhong, Z., Hsieh, T. F., et al. (2018). Robust transcriptional activation in plants using multiplexed CRISPRAct2.0 and mTALE-Act systems. Mol. Plant 11, 245-256. doi: 10.1016/j. molp.2017.11.010

Mikami, M., Toki, S., and Endo, M. (2017). In planta processing of the SpCas9gRNA complex. Plant Cell Physiol. 58, 1857-1867. doi: 10.1093/pcp/pcx154

Mitra, A., Han, J., Zhang, Z. J., and Mitra, A. (2009). The intergenic region of Arabidopsis thaliana cab1 and cab2 divergent genes functions as a bidirectional promoter. Planta 229, 1015-1022. doi: 10.1007/s00425-008-0859-1

Nekrasov, V., Staskawicz, B., Weigel, D., Jones, J. D., and Kamoun, S. (2013). Targeted mutagenesis in the model plant Nicotiana benthamiana using Cas 9
RNA-guided endonuclease. Nat. Biotechnol. 31, 691-693. doi: 10.1038/ nbt. 2655

Poliner, E., Takeuchi, T., Du, Z. Y., Benning, C., and Farre, E. M. (2018). Nontransgenic marker-free gene disruption by an episomal CRISPR system in the Oleaginous Microalga, Nannochloropsis oceanica CCMP1779. ACS Synt. Biol. 7, 962-968. doi: 10.1021/acssynbio.7b00362

Shan, Q., Wang, Y., Chen, K., Liang, Z., Li, J., Zhang, Y., et al. (2013). Rapid and efficient gene modification in rice and Brachypodium using TALENs. Mol. Plant 6, 1365-1368. doi: $10.1093 / \mathrm{mp} / \mathrm{sss} 162$

Stewart, C. N., Jr. and Via, L. E. (1993). A rapid CTAB DNA isolation technique useful for RAPD fingerprinting and other PCR applications. Biotechniques $14,748-750$.

Svitashev, S., Schwartz, C., Lenderts, B., Young, J. K., and Mark Cigan, A. (2016). Genome editing in maize directed by CRISPR-Cas9 ribonucleoprotein complexes. Nat. Commun. 7, 13274. doi: 10.1038/ncomms13274

Tang, X., Liu, G., Zhou, J., Ren, Q., You, Q., Tian, L., et al. (2018). A large-scale whole-genome sequencing analysis reveals highly specific genome editing by both Cas9 and Cpf1 (Cas12a) nucleases in rice. Genome Biol. 19, 84. doi: 10.1186/s13059-018-1458-5

Tang, X., Lowder, L. G., Zhang, T., Malzahn, A. A., Zheng, X., Voytas, D. F., et al. (2017). A CRISPR-Cpf1 system for efficient genome editing and transcriptional repression in plants. Nat. Plants 3, 17018. doi: 10.1038/ nplants.2017.18

Tang, X., Ren, Q., Yang, L., Bao, Y., Zhong, Z., He, Y., et al. (2019). Single transcript unit CRISPR 2.0 systems for robust Cas9 and Cas12a mediated plant genome editing. Plant Biotechnol. J. 17, 1431-1445. doi: 10.1111/pbi.13068

Tang, X., Zheng, X., Qi, Y., Zhang, D., Cheng, Y., Tang, A., et al. (2016). A single transcript CRISPR-Cas9 system for efficient genome editing in plants. Mol. Plant 9, 1088-1091. doi: 10.1016/j.molp.2016.05.001

Tsai, S. Q., Wyvekens, N., Khayter, C., Foden, J. A., Thapar, V., Reyon, D., et al. (2014). Dimeric CRISPR RNA-guided FokI nucleases for highly specific genome editing. Nat. Biotechnol. 32, 569-576. doi: 10.1038/nbt.2908

Wang, R., Yan, Y., Zhu, M., Yang, M., Zhou, F., Chen, H., et al. (2016). Isolation and functional characterization of bidirectional promoters in rice. Front. Plant Sci. 7, 766. doi: 10.3389/fpls.2016.00766

Woo, J. W., Kim, J., Kwon, S. I., Corvalan, C., Cho, S. W., Kim, H., et al. (2015). DNA-free genome editing in plants with preassembled CRISPR-Cas9 ribonucleoproteins. Nat. Biotechnol. 33, 1162-1164. doi: 10.1038/nbt.3389

Xie, K., Minkenberg, B., and Yang, Y. (2015). Boosting CRISPR/Cas9 multiplex editing capability with the endogenous tRNA-processing system. Proc. Natl. Acad. Sci. U. S. A. 112, 3570-3575. doi: 10.1073/pnas.1420294112

Xie, M., He, Y., and Gan, S. (2001). Bidirectionalization of polar promoters in plants. Nat. Biotechnol. 19, 677-679. doi: 10.1038/90296

Xu, R., Qin, R., Li, H., Li, D., Li, L., Wei, P., et al. (2016). Generation of targeted mutant rice using a CRISPR-Cpf1 system. Plant Biotechnol. J. 15, 713-717. doi: $10.1111 /$ pbi.12669

Yang, S., Sleight, S. C., and Sauro, H. M. (2013). Rationally designed bidirectional promoter improves the evolutionary stability of synthetic genetic circuits. Nucleic Acids Res. 41, e33. doi: 10.1093/nar/gks972

You, Q., Zhong, Z., Ren, Q., Hassan, F., Zhang, Y., and Zhang, T. (2018). CRISPRMatch: an automatic calculation and visualization tool for high-throughput CRISPR genome-editing data analysis. Int. J. Biol. Sci. 14, 858-862. doi: 10.7150/ijbs.24581

Zhang, T., Marand, A. P., and Jiang, J. (2016). PlantDHS: a database for DNase I hypersensitive sites in plants. Nucleic Acids Res. 44, D1148-D1153. doi: 10.1093/nar/gkv962

Zhang, Y., Zhang, F., Li, X., Baller, J. A., Qi, Y., Starker, C. G., et al. (2013). Transcription activator-like effector nucleases enable efficient plant genome engineering. Plant Physiol. 161, 20-27. doi: 10.1104/pp.112.205179

Zheng, X., Yang, S., Zhang, D., Zhong, Z., Tang, X., Deng, K., et al. (2016). Effective screen of CRISPR/Cas9-induced mutants in rice by single-strand conformation polymorphism. Plant Cell Rep. 35, 1545-1554. doi: 10.1007/s00299-016-1967-1

Zhong, Z., Sretenovic, S., Ren, Q., Yang, L., Bao, Y., Qi, C., et al. (2019). Improving plant genome editing with high-fidelity $\mathrm{xCas} 9$ and noncanonical PAM-targeting Cas9-NG. Mol. Plant. 12, 1027-1036. doi: 10.1016/j.molp.2019.03.011

Zhong, Z., Zhang, Y., You, Q., Tang, X., Ren, Q., Liu, S., et al. (2018). Plant genome editing using FnCpf1 and LbCpf1 nucleases at redefined and altered pam sites. Mol. Plant 11, 999-1002. doi: 10.1016/j.molp.2018.03.008 
Zhou, J., Deng, K., Cheng, Y., Zhong, Z., Tian, L., Tang, X., et al. (2017). CRISPR-Cas9 based genome editing reveals new insights into microRNA function and regulation in rice. Front. Plant Sci. 8, 1598. doi: 10.3389/ fpls.2017.01598

Zhou, J., Xin, X., He, Y., Chen, H., Li, Q., Tang, X., et al. (2019). Multiplex QTL editing of grain-related genes improves yield in elite rice varieties. Plant Cell Rep. 38, 475-485. doi: 10.1007/s00299-018-2340-3

Zhu, B., Zhang, W., Zhang, T., Liu, B., and Jiang, J. (2015). Genome-wide prediction and validation of intergenic enhancers in Arabidopsis using open chromatin signatures. Plant Cell 27, 2415-2426. doi: 10.1105/ tpc. 15.00537
Conflict of Interest Statement: The authors declare that the research was conducted in the absence of any commercial or financial relationships that could be construed as a potential conflict of interest.

Copyright (C) 2019 Ren, Zhong, Wang, You, Li, Yuan, He, Qi, Tang, Zheng, Zhang, Qi and Zhang. This is an open-access article distributed under the terms of the Creative Commons Attribution License (CC BY). The use, distribution or reproduction in other forums is permitted, provided the original author(s) and the copyright owner(s) are credited and that the original publication in this journal is cited, in accordance with accepted academic practice. No use, distribution or reproduction is permitted which does not comply with these terms. 